\title{
Hernioplastia inguinal con técnica de liechtenstein: índice de recidivas, seguimiento a 36 meses en el servicio de cirugía general del hospital regional de Pilar
}

\author{
Pedro Mariano Pintos Fernández
}

Mariano.9023@hotmail.com

María José Benítez Noguera majobetnog@gmail.com

Anna Fiorella Daiub Alviso Fiodaiub777@,hotmail.es

Gabrielle Beleni Daiub Alviso

RESUMEN

Belga453@gmail.com

La hernioplastia sin tensión de Lichtenstein es una de las técnicas más aceptadas como método de reparación en la hernia inguinal. Se realizó un estudio retrospectivo y observacional donde se efectuó hernioplastia inguinal con técnica de Lichtenstein a 30 pacientes con hernia inguinal en el Hospital regional de Pilar durante el año 2018. El objetivo fue determinar la efectividad de dicha técnica mediante el índice de recidivas. El seguimiento a los pacientes se realizó a los 7, 14 días posteriormente a los 1, 6, 12 , 18, 24 y 36 meses. La edad media de los pacientes fue de 51 años y un $90 \%$ del sexo masculino, la procedencia de los pacientes fue $78 \%$ urbana. El tiempo quirúrgico promedio fue de $48 \mathrm{~min}$. En relación a la estancia hospitalaria el $90 \%$ de los pacientes recibió el alta a las 24 horas. Los tipos de hernias reparadas según la clasificación de Gilbert el tipo I fue el más frecuente con un 40\%. El índice de recidivas fue de 3,33\%. Las complicaciones fueron; Infección del sitio quirúrgico, Seroma y la Inguinodinia. El principal indicador utilizado para evaluar los resultados de la hernioplastia inguinal sigue siendo el índice de recidiva. En la actualidad la mayoría de los cirujanos eligen técnicas sin tensión de lichtenstein presentar bajo índice de recidiva, ser simple y rápida, menor tiempo de hospitalización post cirugía, baja morbilidad.

Palabras clave: hernioplastia inguinal; técnica de lichtenstein; recidivas; efectividad 


\title{
Inguinal hernioplasty with the liechtenstein technique: recurrence rate, 36-month follow-up in the general surgery service of the regional hospital of Pilar
}

\begin{abstract}
The Lichtenstein tension-free hernioplasty is one of the most accepted techniques as a repair method for inguinal hernia. A retrospective and observational study was carried out in which inguinal hernioplasty with the Lichtenstein technique was performed in 30 patients with inguinal hernia at the Regional Hospital of Pilar during 2018. The objective was to determine the effectiveness of this technique by means of the recurrence rate. The patients were followed up at 7,14 days later at 1, 6, 12, 18, 24 and 36 months. The mean age of the patients was 51 years and $90 \%$ of the male sex, the origin of the patients was $78 \%$ urban. The average surgical time was $48 \mathrm{~min}$. Regarding the hospital stay, $90 \%$ of the patients were discharged within 24 hours. The types of hernias repaired according to Gilbert's classification, type I was the most frequent with $40 \%$. The recurrence rate was $3.33 \%$. The complications were; Surgical site infection, Seroma and Inguinodynia. The main indicator used to evaluate the results of inguinal hernioplasty continues to be the recurrence rate. Currently, most surgeons choose techniques without Lichtenstein tension, presenting a low rate of recurrence, being simple and fast, shorter post-surgery hospitalization time, and low morbidity.
\end{abstract}

Key words: Inguinal hernioplasty, Lichtenstein technique, Recurrences, effectiveness 


\section{INTRODUCCION}

La hernia inguinal se define como la protrusión o salida ocasional o permanente, de una víscera o tejido a través de un orificio o defecto de la pared abdominal, anatómicamente constituido. (Ferraina 2003).

Las hernias inguinales pueden aparecer en cualquier momento de la vida, a cualquier edad y presentarse en ambos sexos, pudiendo ser unilaterales o bilaterales y se clasifican de acuerdo con los hallazgos quirúrgicos en varios tipos, pudiendo repararse mediante diferentes técnicas quirúrgicas empleadas todas ellas bajos riesgos o complicaciones a corto, mediano y largo plazo. Las complicaciones locales pueden aparecer a corto plazo como hematoma, neuralgia, Seroma, orquitis isquémica e infección de la herida operatoria . (Hidalgo et ál., 2001)

Las hernias inguinales se manifiestan por un aumento del volumen en la región inguinal por protrusión del saco herniario y pueden clasificarse en hernias directas, indirectas, además pueden ser mixtas y estar acompañadas de hernias femorales. (Carrera-Laureán et ál., 2018)

La clasificación de las hernias inguinales según Gilbert que fue publicada en 1989, basadas en consideraciones anatómicas y funcionales, como describe 5 tipos de hernias. A estos tipos, Rutkow y Robbins añadieron a esta clasificación 2 tipos más en el año 1993 quedando así las Clasificación de modificada por Rutkow y Robbins. (RomeroVargas et át 2018).

Esta clasificación fue modificada en 1993 por Rutkow y Robbins añadiendo dos nuevas categorías.

La hernia inguinal es un problema de salud frecuente y su reparación constituye el procedimiento quirúrgico más frecuente para el cirujano general. La probabilidad de desarrollar una hernia inguinal, en nuestra vida, es en varón un $27 \%$ frente al $3 \%$ en la mujer. Las técnicas quirúrgicas se pueden clasificar en tres grupos: Técnicas abiertas en las que se pueden emplear material protésico (Lichtenstein, Gilbert, Rutkow- Robbins) o no (Bassini, Shouldice, McVay, etc) y técnicas laparoscópicas protésicas. La técnica de Lichtenstein se considera el gold standar para la reparación de la hernia inguinal primaria. (Turiño-Luque et ál 2018).

Aunque la obesidad es una condición clínica individual, se ha convertido en un grave problema de salud pública que está aumentando en proporciones epidémicas en todo el 
mundo. Dado la alta incidencia y prevalencia de esta enfermedad, que conduce a un gran impacto económico y social de esta enfermedad. Se sabe que los obesos que deben someterse a una cirugía sufren una mayor tasa de complicaciones cardiovasculares, infección del sitio quirúrgico, entre otras. Aunque los estudios muestran que la tasa de mortalidad no varía entre pacientes obesos y no obesos, la mortalidad es mucho mayor en pacientes con obesidad mórbida.

Las complicaciones postoperatorias son los principales desafíos para costos de salud y / o atención médica para pacientes obesos. Respecto a el procedimiento quirúrgico y la presencia de sobrepeso estudios anteriores han informado que los pacientes con sobrepeso u obesidad tienen resultados quirúrgicos adversos que incluyen mayor tiempo quirúrgico y mayor tiempo de estadía hospitalaria (Faes- Petersen et ál; 2016).

La hernioplastia sin tensión de Lichtenstein es en la actualidad una de las técnicas más aceptadas como método de reparación en hernia inguinal, debido a su efectividad, bajo índice de recidiva y fácil reproducibilidad. Sin embargo, no está exenta de problemas postoperatorios como dolor e irritación local crónica atribuidos a las suturas para la fijación de la malla (Rocha-G. 2008).

Este tipo de cirugías son realizadas en su mayoría por médicos residentes en entrenamiento, siempre bajo la supervisión de médicos especialistas en hernias, en estudios similares las cirugías realizadas por herniólogos expertos son las que tienen índices de recidiva menores al 1\%, en algunas hernioplastias cuando la cirugía es realizada por cirujanos no herniólogos la recidiva puede ir desde el 1 al 3\%. (Respardo et ál; 2014). Se considera al índice de recidiva o recurrencia como el mejor indicador de la efectividad de la técnica quirúrgica.

El objetivo del presente estudio fue determinar la efectividad de dicha técnica midiendo el índice de recidivas, características socio-demográficas, co-morbilidades y la clasificación de las hernias según Gilbert modificado por Rutkow y Robbins.

\section{MATERIALES Y METODOS}

Se realizó un estudio descriptivo, retrospectivo y observacional donde se evaluaron los resultados de 30 procedimientos quirúrgicos a pacientes que padecían hernia inguinal y a los que se practicó hernioplastia libre de tensión con la técnica de Lichtenstein en el Hospital Regional de Pilar durante el año 2018 a los cuales se le realizó un seguimiento por 36 meses posterior a la intervención quirúrgica. Se evaluaron los datos 
demográficos como sexo, edad, lugar de procedencia, tiempo quirúrgico en minutos, estancia hospitalaria en horas, en el seguimiento de los pacientes donde se evaluó el índice de recidiva. Se evaluó el estado nutricional de los pacientes en el pre operatorio y fueron medidas las variables peso $(\mathrm{kg})$ y talla $(\mathrm{m})$, para determinar si existe sobrepeso y obesidad se estableció el índice de masa corporal (IMC) de cada paciente según la formula peso en kilogramos dividido por la talla en metros al cuadrado. Los puntos de corte utilizados para la clasificación del Índice de Masa Corporal fueron los propuestos por la Organización Mundial de la Salud (OMS), que define sobrepeso cuando el IMC es igual o superior a $25 \mathrm{~kg} / \mathrm{m} 2$ y obesidad cuando es superior a $30 \mathrm{~kg} / \mathrm{m} 2$.

Los pacientes fueron seguidos por consulta externa durante un período de 7 y 14 días posteriormente a $\operatorname{los} 1,6,12,18,24$ y 36 meses. En esta serie los pacientes se realizaron cirugías electivas y programadas. La técnica quirúrgica que se utilizó para la reparación de las hernias inguinales fue la de Lichtenstein.

Los datos demográficos y clínicos se obtuvieron de las fichas clínicas y se procesaron con el programa de Excel. Los resultados se exponen en tablas y figuras. Los criterios de inclusión fueron, pacientes mayores de edad, de ambos sexos, con diagnóstico de hernia inguinal, haber sido intervenido quirúrgicamente de hernioplastía inguinal abierta con técnicas sin tensión de Lichtenstein dentro del periodo 2018 en el Servicio de Cirugía del Hospital Regional de Pilar. Los pacientes excluidos son aquellos con hernias inguinales en las cuales se utilizaron otras técnicas quirúrgicas, pacientes cuya información en los expedientes eran incompletas y pacientes perdidos durante el seguimiento. Se respetaron todas las normas éticas, manteniéndose así todos los datos de los pacientes en total confidencialidad.

Los Autores declaran no tener conflicto de intereses en el presente trabajo.

No se recibió ningún tipo de financiación o ayuda externa para el presente trabajo

\section{RESULTADOS}

Se realizaron 30 intervenciones quirúrgicas donde se procedió a reparar las hernias inguinales con técnica sin tensión de Lichtenstein. El índice de recidivas fue de 3,33\%. La población tuvo una edad promedio de 51 años con mínimo de 27 y máximo de 79 años. Del total de los pacientes 26 fueron del sexo masculino (90\%) y solo 4 (10\%) del sexo femenino. La procedencia de los pacientes fue $78 \%$ Urbana y $22 \%$ Rural. 
El tiempo quirúrgico promedio fue de 48 min con un mínimo de 40 min y un máximo de 75 min.

La localización de la hernia se identificó en el lado derecho en el 53,3\% de los casos; en el lado izquierdo en el 46,7\%.

En relación a la estancia hospitalaria postoperatoria de los pacientes, el promedio de la estancia fue de 26 horas, donde el $90 \%$ recibió el alta a las 24 horas, los demás datos se muestran en la Tabla 1.

\begin{tabular}{|c|c|c|}
\hline \multicolumn{2}{|c|}{ Tabla 1. Estancia hospitalaria de los pacientes post operados de hernioplastia } \\
inguinal $n=30$
\end{tabular}

Fuente: archivo de fichas clínicas Hospital Regional de Pilar

El estado nutricional de los pacientes evaluados según IMC se muestra en la tabla 2.

Se observó que el que el $62 \%$ de los pacientes tenía sobrepeso y $13 \%$ obesidad

\begin{tabular}{lcc}
\hline & Tabla 2. Estado nutricional según IMC (OMS 2007) & $n=30$ \\
\hline Peso normal & $18,5-24,9 \mathrm{Kg} / \mathrm{m}^{2}$ & $25 \%(5)$ \\
Sobrepeso & $25,0-29,9 \mathrm{Kg} / \mathrm{m}^{2}$ & $62 \%(19)$ \\
Obesidad & $\geq 30,0 \mathrm{Kg} / \mathrm{m}^{2}$ & $13 \%(6)$ \\
\hline
\end{tabular}

Fuente: fichas clínicas- Servicio Cirugía Hospital Regional de Pilar

Con respecto al hábito de fumar, se encontrón un $23 \%$ de los pacientes eran fumadores y un $77 \%$ no fumadores.

Los tipos de hernias reparadas con la técnica de lichtenstein encontradas en el estudio fueron agrupadas según la clasificación de Gilbert Modificada donde observamos un predominio de las hernias indirectas de Tipo I y II las cuales se muestran en la Tabla 3 


\begin{tabular}{|c|c|c|}
\hline \multicolumn{2}{|c|}{ Tabla 3: Tipos de hernias reparadas según Clasificación de Gilbert Modificada } \\
$(N=30)$
\end{tabular}

Fuente: Fichas clínicas - Servicio de Cirugía General- Hospital Regional De Pilar

Para evaluar la eficacia de la técnica se realizó el seguimiento de los pacientes donde se encontró la recidiva de solo un paciente representando así el $3.33 \%$ que se constató a los 24 meses del seguimiento.

\section{DICUSION - CONCLUSION}

Las hernias son un problema frecuente de salud que afecta a ambos sexos en todas las edades. (Dumenigo et ál, 2007).

La definición de recurrencia o recidiva se asume como la reaparición de un defecto herniario en el mismo sitio anatómico específico de la hernia primariamente operada después de una desaparición quirúrgica temporal; de otro modo, se tratará de una segunda hernia primaria y diferente (Fonseca, F.K., ét al)

El principal indicador utilizado para evaluar los resultados de la hernioplastia inguinal sigue siendo el índice de recidiva. Con la técnica de Lichtenstein donde preconizó la hernioplastía libre de tensión, la recurrencia reportada ha sido hasta menor al $1 \%$ (Respardo-Ramírez et ál, 2014) en nuestro estudio hemos tenido una recidiva próxima a la reportada en la literatura con el $3.33 \%$. Teniendo en cuenta este resultado cercano a lo reportado por la literatura.

En cuanto al género como es de esperarse las hernias inguinales se presentan más en hombres que en mujeres coincidiendo con un estudio de Bruna et ál (2014) donde el 
91,1\% fueron del sexo masculino, siendo las edades promedio de los pacientes de 60 años, datos semejantes al de nuestro estudio.

Con respecto al tiempo quirúrgico un estudio de Bernal et ál (2014) encontró que el tiempo promedio quirúrgico fue de 45 min semejante al de nuestro equipo.

La hernia inguinal se encuentra con más frecuencia en los pacientes del género masculino con edades comprendidas entre la cuarta y la séptima década de la vida. Las hernias de tipo I y II de la clasificación de Gilbert modificada por Rutkow y Robbins es el más frecuente encontrado en este estudio, parcialmente similiar a la encontrada por Respardo Ramirez y col (2014) que reporto los tipos II y III como los más frecuentes. Con relación a la estancia Hospitalaria en estudios similares al nuestro se encontró un tiempo de alta más frecuente de 24 horas similar a la de este estudio cuyo promedio fue de 26 horas.

Entre las co-morbilidades estudiadas el sobrepeso estuvo presente en el $62 \%$ y la obesidad en el $13 \%$.

Existe evidencia que demuestra una mayor frecuencia de hernias inguinales en fumadores, al compararlos con la población sin el hábito de fumar. (Borquez ét al 2005) con respecto al hábito de fumar de los pacientes se encontró 14 pacientes fumadores de los cuales 5 de esos pacientes presentaron una complicación post quirúrgica.

En la actualidad la mayoría de los cirujanos eligen técnicas sin tensión de lichtenstein por presentar bajo índice de recidivas, ser simple y rápida, menor tiempo de hospitalización post cirugía, baja morbilidad.

\section{REFERENCIAS BIBLIOGRAFICAS}

Adorno, A., Monges, L., Lezcano, M. J., Cuenca, Osmar, B., José, \& Ramírez, J. (2017). Abordaje Laparoscópico Tapp De Las Hernias Inguinales. Experiencia Inicial Hospital Nacional. Cirugía paraguaya,4l(1), 1417. https://dx.doi.org/10.18004/sopaci.abril.14-17

Bernal-Gómez, R.,, Sagüi de la Fuente, L., Olivares Ontiveros, O., Olivares Valdez O. (2014) Plastia inguinal tipo Lichtenstein versus reparación inguinal laparoscópica TAPP. Ensayo clínico abierto Rev Cir Gen, 3(1): 9-14 http//www.elsevier.es/cirujanogeneral

Bórquez P, Garrido L, Peña P. Fisiopatología de hernia primaria, algo más que un fenómeno mecánico. (2005). Rev. Chilena de Cirugía 57(5): 432-435. 
Bruna-Esteban, M., Cantos-Pallarés, M., Antigues Sánchez de Rojas, E., Vila, M.J. (2014) Estudio prospectivo y aleatorizado de los resultados a largo plazo de la hernioplastia. Rev Cir Esp 92(3): 195-200

Carrasco-Sanchez, F.J., Diaz-Alcaide, F., Marín-Fernández, Y., Chaparro-Moreno I., Pujol-de la Llave E., (2012) Prevalencia de obesidad en pacientes médicos hospitalizados. An Med Interna (Madrid) 19: 453-6.

Carrera-Laurean, N., Camacho-López, M.R. (2018) Tipo de hernia inguinal encontrado en la población general, según la clasificación de Nyhus. Rev. Mexicana $\begin{array}{llll}\text { CIRUJANO } & \text { GENERAL; }\end{array}$ Htpp://www.mediagraphic.com/cirujanogeneral

Disponible

en:

http://hospital.uas.edu.mx/revmeduas/pdf/v4/n2/Hernioplastia\%20con\%20tecnica\% 20de\%20Lichtenstein.pdf

Duménigo-Arias, O. , de Armas-Pérez, B. , Martínez Ferrá, G. , \& Gil Hernández, Ailette. (2007). Hernioplastia inguinal de Lichtenstein: la mejor opción. Revista Cubana de Cirugía, 46(1) Recuperado en 29 de marzo de 2020, de http://scielo.sld.cu/scielo.php?script=sci_arttext\&pid=S0034$74932007000100007 \& \operatorname{lng}=$ es\&tlng=es.

Enríquez-Weinmann E.S., Roselló-Fina J.R., Canals-Rabassa P.P., Mederos-Curbelo O.N., Morejón-Pozo C. (2003) Reparación protésica de hernias inguinales con técnica de Lichtenstein. Revista Cubana de Cirugía; 43(1):28-33. Recuperado en 9 de mayo de 2020, de http://scielo.sld.cu/scielo.php?script=sci_arttext\&pid=S0034$\underline{74932003000100005 \& \operatorname{lng}=\text { es\&tlng=es. }}$

Faes-Petersen, R., Diaz-Giron-Gidi, A., Velez-Perez, F., Gonzalez-Chavez, M.A., Lemus, R., Corea-Rovelo, J.M., Villegas-Tovar, e., et (2016 january- march), Overweight and obesity as a risk factor for postoperative complications in patiets undergoing inguinal hernia repair, cholecystectomy and appendectomy. Rev Invest Med Sur Mex, 23(1):28-33

Fonseca S., Fernando Karel, Lorenzo M., Andrés, Rey V., Yaima Susana, \& Llópiz S., Rolando. (2019). Factores de riesgo asociados a la hernia inguinal recurrente en el adulto mayor. Revista de cirugía, 71(1), 61-65. https://dx.doi.org/10.4067/S245245492019000100061 
Gilbert AI. An anatomic and functional classification for the diagnosis and treatment of inguinal hernia. a.m. J Surg. 1989 Mar; 157(3): 331-333.

Herrera-Gutierrez, L., Roldan-Aviña, J.P., Muñoz-del Pozo, F., Palacios-Garcia, E. (2018 mayo) Comepndio de las clasificaciones anatómicas de las hernias de la pared abdominal y validez actual de las mismas. Rev Cir And ;29(2):74-76; disponible

en: https://www.asacirujanos.com/admin/upfiles/revista/2018/Cir_Andal_vol29_n2_act ualizacion1.pdf

Hidalgo, M., Castellón, C., Figueroa, J.M., Eymar, J.L., González, E.M. (2001) Complicaciones de la cirugía de las hernias. Rev Cir Esp., 69, 217-223.

Lozano-Corona R, Beltran-Estrada A, Gómez-Rios N, Rodriguez-Ortega F. (2012) Irving Lichtenstein, maestro de la Cirugia Inguinal, semblanza de su vida y obra. Rev. Cirujano General 34(1); 72-77

Organización Mundial de la Salud. Obesidad y sobrepeso. OMS; 2017; Available from: https://www.who.int/features/factfiles/obesity/es/

Respardo-Ramírez, C.A., Quiñónez-Meza, M.E., Peraza-Garay, F., Ortiz-Bojórquez, J.C., Astorga-Ortiz, M.S., Pérez-León, B., Esquivel-Leyva, B.I., (2014 abril-junio) Hernioplastía inguinal con técnica de Lichtenstein: incidencia de recidiva en seguimiento a cinco años Rev Med UAS ; 4(2): 43-47

Rocha-G, M. (2008 abril). Hernioplastia inguinal con malla sin tensión: Experiencia con adhesivo N-butil-cianoacrilato. Revista chilena de cirugía,60(2); 98102. https://dx.doi.org/10.4067/S0718-40262008000200003

Rodríguez-Ortega MF, Cárdenas-Martínez G, López-Castañeda H.(2003) Evolución histórica del tratamiento de la hernia inguinal. Cir Cir; 71: 245-251.

Sachs M, Damm M, Encke A. (1997) Historical evolution of inguinal hernia repair.

World J Surg; 21: 218-223

Samaniego-Ayala,C., Janzen Enns, D., (2010) Hernioplastia inguinal electiva. An. Fac.

Cien. Med. (Asunción), 43(2): 49-55 disponible en: http://scielo.iics.una.py/pdf/anales/v43n2/v43n2a06.pdf

Turiño-Luque J.D., Mirón-Fernandez, I., Rivas-Becerra, J., Cabello-Burgos, A.J, Rodriguez-Silva, C., Martinez-FFerriz, A., Santoyo-Santoyo, J. (2018 mayo) Hernioplastía ingunal abierta: Técnica de Lichtensteins. Cir Andal.; 29(2); 160-162. 